\title{
Notification List Notification that new names and new combinations
have appeared in volume 60 , part 8 , of the IJSEM
}

Correspondence

Jean Euzéby

email address can be found at www.bacterio.net
This listing of names published in a previous issue of the IJSEM is provided as a service to bacteriology to assist in the recognition of new names and new combinations. This procedure was proposed by the Judicial Commission [Minute 11(ii), Int J Syst Bacteriol 41 (1991), p. 185]. The names given herein are listed according to the Rules of priority (i.e. page number and order of valid publication of names in the original articles). Taxonomic opinions included in this List (i.e. the creation of synonyms or the emendation of circumscriptions) cannot be considered as validly published nor, in any other way, approved by the International Committee on Systematics of Prokaryotes and its Judicial Commission.

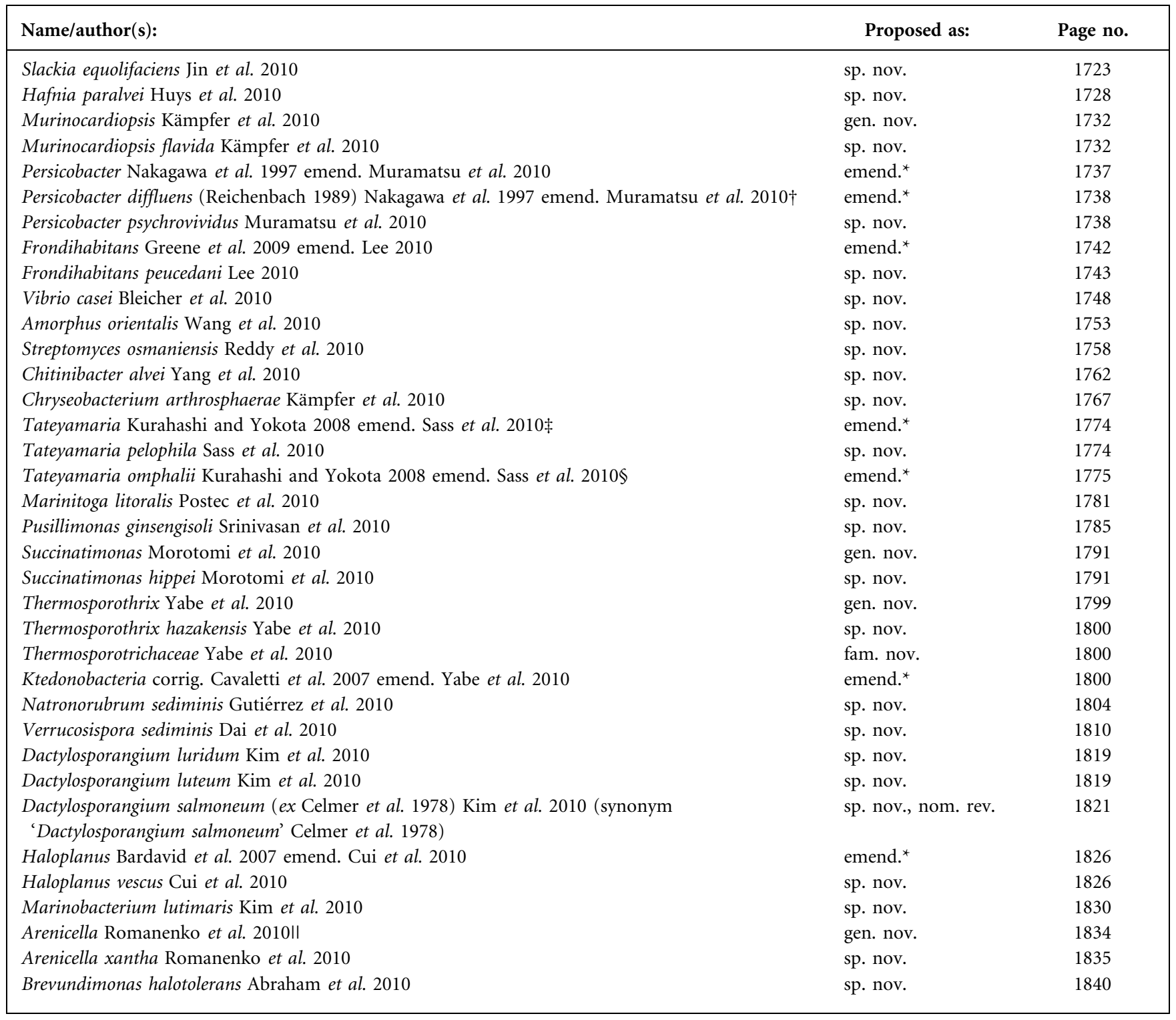


cont.

\begin{tabular}{|c|c|c|}
\hline Name/author(s): & Proposed as: & Page no. \\
\hline Brevundimonas poindexterae Abraham et al. 2010 & sp. nov. & 1841 \\
\hline Brevundimonas staleyi Abraham et al. 2010 & sp. nov. & 1841 \\
\hline Haliea mediterranea Lucena et al. 2010 & sp. nov. & 1847 \\
\hline Parapedobacter luteus Kim et al. 2010 & sp. nov. & 1851 \\
\hline Parapedobacter composti Kim et al. 2010 & sp. nov. & 1852 \\
\hline Saccharomonospora marina Liu et al. 2010 & sp. nov. & 1856 \\
\hline Marivirga Nedashkovskaya et al. 2010 & gen. nov. & 1860 \\
\hline $\begin{array}{l}\text { Marivirga sericea (ex Lewin 1969) Nedashkovskaya et al. } 2010 \text { (synonym 'Microscilla sericea' } \\
\text { Lewin } 1969\end{array}$ & nom. rev., comb. nov. & 1860 \\
\hline $\begin{array}{l}\text { Marivirga tractuosa (Lewin 1969) Nedashkovskaya et al. } 2010 \text { [basonym Flexibacter tractuosus } \\
\text { (Lewin 1969) Leadbetter } 1974 \text { (Approved Lists 1980)] }\end{array}$ & comb. nov. & 1861 \\
\hline Bacteroides clarus Watanabe et al. 2010 & sp. nov. & 1867 \\
\hline Bacteroides fluxus Watanabe et al. 2010 & sp. nov. & 1868 \\
\hline Bacteroides oleiciplenus Watanabe et al. 2010 & sp. nov. & 1868 \\
\hline Campylobacter volucris Debruyne et al. 2010 & sp. nov. & 1873 \\
\hline Chryseobacterium solincola Benmalek et al. 2010 & sp. nov. & 1879 \\
\hline Kushneria sinocarnis Zou and Wang 2010 & sp. nov. & 1884 \\
\hline Francisella Dorofe'ev 1947 (Approved Lists 1980) emend. Huber et al. 2010 & emend. ${ }^{*}$ & 1894 \\
\hline Francisella hispaniensis Huber et al. 2010 & sp. nov. & 1895 \\
\hline $\begin{array}{l}\text { Francisella tularensis subsp. novicida (Larson et al. 1955) Huber et al. } 2010 \text { [basonym } \\
\text { Francisella novicida (Larson et al. 1955) Olsufiev et al. } 1959 \text { (Approved Lists 1980)] }\end{array}$ & comb. nov. & 1895 \\
\hline Agrococcus terreus Zhang et al. 2010 & sp. nov. & 1902 \\
\hline Micrococcus terreus Zhang et al. 2010 & sp. nov. & 1902 \\
\hline Saccharopolyspora phatthalungensis Duangmal et al. 2010 & sp. nov. & 1907 \\
\hline Paenibacillus glacialis Kishore et al. 2010 & sp. nov. & 1912 \\
\hline Lactobacillus pobuzihii Chen et al. 2010 & sp. nov. & 1916 \\
\hline Ruegeria pelagia Lee et al. 2007 pro synon. Ruegeria mobilis Muramatsu et al. 2007 & synon. ${ }^{\star}$ & 1919 \\
\hline Xenorhabdus vietnamensis Tailliez et al. 2010 & sp. nov. & 1933 \\
\hline Photorhabdus temperata subsp. khanii Tailliez et al. 2010 & subsp. nov. & 1935 \\
\hline Photorhabdus temperata subsp. tasmaniensis Tailliez et al. 2010 & subsp. nov. & 1935 \\
\hline Photorhabdus luminescens subsp. hainanensis Tailliez et al. 2010 & subsp. nov. & 1935 \\
\hline Photorhabdus luminescens subsp. caribbeanensis Tailliez et al. 2010 & subsp. nov. & 1935 \\
\hline $\begin{array}{l}\text { Photorhabdus temperata subsp. thracensis (Hazir et al. 2004) Tailliez et al. } 2010 \text { (basonym } \\
\text { Photorhabdus luminescens subsp. thracensis Hazir et al. 2004) }\end{array}$ & comb. nov. & 1935 \\
\hline Massilia jejuensis Weon et al. 2010 & sp. nov. & 1941 \\
\hline Naxibacter suwonensis Weon et al. 2010 & sp. nov. & 1942 \\
\hline Corynebacterium marinum Du et al. 2010 & sp. nov. & 1946 \\
\hline Winogradskyella pacifica Kim and Nedashkovskaya 2010 & sp. nov. & 1951 \\
\hline Colwellia asteriadis Choi et al. 2010 & sp. nov. & 1956 \\
\hline Neptunomonas antarctica Zhang et al. 2010 & sp. nov. & 1960 \\
\hline
\end{tabular}

*Taxonomic opinion.

†In the paper by Muramatsu et al. 2010, Persicobacter diffluens is erroneously cited as Persicobacter diffluens Nakagawa et al. 1997.

$¥$ In the paper by Sass et al. 2010, the genus Tateyamaria is erroneously cited as Tateyamaria Kurahashi and Yokota 2007.

\$In the paper by Sass et al. 2010, the species Tateyamaria omphalii is erroneously cited as Tateyamaria omphalii Kurahashi and Yokota 2007. IIOn page 1834, the title of the protologue is erroneously cited as 'Description of Arenicella xantha gen. nov.'. 